\title{
Taking a Lesson From Patients' Recovery Strategies to Optimize Training During Robot-Aided Rehabilitation
}

\author{
Roberto Colombo, Senior Member, IEEE, Irma Sterpi, Alessandra Mazzone, Carmen Delconte, and Fabrizio Pisano
}

\begin{abstract}
In robot-assisted neurorehabilitation, matching the task difficulty level to the patient's needs and abilities, both initially and as the relearning process progresses, can enhance the effectiveness of training and improve patients' motivation and outcome. This study presents a Progressive Task Regulation algorithm implemented in a robot for upper limb rehabilitation. It evaluates the patient's performance during training through the computation of robot-measured parameters, and automatically changes the features of the reaching movements, adapting the difficulty level of the motor task to the patient's abilities. In particular, it can select different types of assistance (time-triggered, activity-triggered, and negative assistance) and implement varied therapy practice to promote generalization processes. The algorithm was tuned by assessing the performance data obtained in 22 chronic stroke patients who underwent robotic rehabilitation, in which the difficulty level of the task was manually adjusted by the therapist. Thus, we could verify the patient's recovery strategies and implement task transition rules to match both the patient's and therapist's behavior. In addition, the algorithm was tested in a sample of five chronic stroke patients. The findings show good agreement with the therapist decisions so indicating that it could be useful for the implementation of training protocols allowing individualized and gradual treatment of upper limb disabilities in patients after stroke. The application of this algorithm during robot-assisted therapy should allow an easier management of the different motor tasks administered during training, thereby facilitating the therapist's activity in the treatment of different pathologic conditions of the neuromuscular system.
\end{abstract}

Index Terms-Motor recovery, neurorehabilitation, robotic therapy, stroke, training optimization.

\section{INTRODUCTION}

$\mathbf{M}$ AJOR advances have occurred in the past 20 years in the development and testing of interventions for stroke rehabilitation [1]. Longitudinal studies on impairment recovery

Manuscript received September 28, 2011; revised January 12, 2012; accepted March 28, 2012. Date of current version May 18, 2012. This work was supported in part by the EU-Research project "HUman behavioral Modeling for enhancing learning by Optimizing hUman-Robot interaction." (HUMOUR) EU grant FP7ICT-231724 and in part by the EU- Cost Action TD1006.

R. Colombo is with the "Salvatore Maugeri" Foundation, IRCCS, Service of Rehabilitation Engineering, 27100 Pavia, Italy and "Salvatore Maugeri" Foundation, IRCCS, Service of Bioengineering, 28010 Veruno (NO), Italy (e-mail: roberto.colombo@fsm.it).

I. Sterpi is with the "Salvatore Maugeri" Foundation, IRCCS, Service of Rehabilitation Engineering, 27100 Pavia, Italy (e-mail: irma.sterpi@fsm.it)

A. Mazzone is with the "Salvatore Maugeri" Foundation, IRCCS, Service of Bioengineering, 28010 Veruno (NO), Italy (e-mail: alessandra.mazzone@fsm. it).

C. Delconte and F. Pisano are with the "Salvatore Maugeri" Foundation, IRCCS, Division of Neurology, 28010 Veruno (NO), Italy (e-mail: carmen.delconte@fsm.it; fabrizio.pisano@fsm.it).

Digital Object Identifier 10.1109/TNSRE.2012.2195679 after stroke suggest that only $33 \%-70 \%$ of patients recover the use of their impaired arm [2], [3]. However, the fact that in spontaneous recovery most of the variance in impairment at six months $(>86 \%)$ is explained by the level of impairment at 30 days could suggest that, independently of the specific therapy delivered to the patient, the impact of rehabilitation is limited in the following five months [4]. For this reason, clinicians have turned their attention to new technologies such as robotic neurorehabilitation that, thanks to its capacity to deliver high dosage and high intensity training protocols, has the potential for a greater impact on impairment as demonstrated by the recent literature both in subacute and chronic patients [5]-[9].

Computational motor learning principles may provide a framework for the design and implementation of optimal rehabilitation protocols [10]. Repetitive practice of an exercise is necessary to increase muscle strength and train coordination of the muscular synergies that move limb segments [11]. In order to maximize outcome, the patient's motivation should be sustained during exercise repetition and the assistance provided by the robotic device should be matched to the patient's abilities. In addition, patients need to be able to practice motor actions in different task and environmental contexts, in order to develop motor schemata that are versatile enough to meet the situations they encounter in daily life [12].

Motivation is an important factor in rehabilitation and is frequently used as a determinant of rehabilitation outcome [13]. In particular, active engagement in a treatment/training intervention is usually equated with motivation, and passivity with lack of motivation. The difficulty level of the motor task, the patient's subjective awareness of the global performance obtained, and the quantity and quality of feedbacks presented during training can influence patient motivation and produce different ways of acting and different performances. The last 10 years of experience with robot-assisted rehabilitation have shown that continuous challenging and assisting can yield substantial advantages in the process of motor learning and improve motor coordination [14].

Several robot control strategies have been implemented to assist patients during exercise of both upper and lower limbs [5], [15]-[17]. Performance-based progressive training schemes have been proposed as a way to gradually reduce the amount of guidance during training. Bell et al. proposed a performance-based progressive guidance scheme for self-learning of a computer-based radar tracking simulation task, which showed significant beneficial effects [18]. A performance-based progressive robot-assisted therapy for stroke patients was first proposed by Krebs et al. [19] in the field of neurorehabilitation. 
In Krebs' study, the patients were provided with guidance during a reaching task. In particular a specific algorithm used the mean velocity and the deviation of the patient's motion from a normal movement trajectory to trigger and change the amount of guidance. Similarly, in another robot-assisted rehabilitation study for gait training, human motor adaptation to dynamic environments was modelled as a kinematic error (step height error) corrective learning process and the control gains of the guidance robot were adjusted at each trial based on the error [20]. The results of this study suggested that providing guidance only when needed is more effective than always assisting with a fixed amount. O'Malley et al. demonstrated that a progressive control guidance scheme reduces the dependency of participants on guidance by adjusting the control gains based on individual participant performance. In particular, a progressive shared control algorithm was applied to expose subjects to an appropriate amount of haptic guidance based on their performance [21], [22].

Basically, progressive control should permit the difficulty of the task to increase while at the same time assistance is gradually reduced. Algorithms adapting the difficulty level of the task have been proposed in applications of virtual reality technologies both for motor learning assessment and neurorehabilitation [23], [24]. Such an algorithm was included also in the ADAPT device, a robot that provides adaptive and automatic presentation of tasks by selecting different tools of an occupational therapy exercise task, based on the patient's performance. It does not include any limb support and mechanisms of assistance [25]. To our knowledge, current robotic devices providing assistance or associated control algorithms include different tasks and games but do not offer the possibility of automatically changing the type of motor task administered or other features of the task in order to maintain a high level of patient involvement, and hence motivation, throughout the whole course of treatment.

In a recent paper, we demonstrated that movements executed outside the training workspace were slower, less accurate, and less smooth than those within. Therefore, generalization of motor recovery can only be expected on movements falling within the workspace spanned by the movements executed during training [26].

In order to maximize patient motivation and promote generalization processes through varied therapy practice, we developed a progressive task regulation (PTR) algorithm able to evaluate the patient's performance during training and automatically change the features of the reaching movements (type of assistance and task), so as to adapt the difficulty level of the motor task to the patient's performance and ability. In addition we verified if the results obtained in the assessment of the time course of recovery of chronic poststroke patients could be useful to tune the implementation of the algorithm.

The aim of this paper is to present the algorithm, its design and implementation strategies and describe its application with previously recorded patient's performance data and in some chronic stroke patients in which the algorithm conditioned the patients' behavior ("closed loop" condition). This paper is organized as follows: the recovery strategy hypothesis section describes the basic idea underlying the performance metrics used to design the algorithm; the methods describe in detail the features of the tasks used to implement the different difficulty levels and the rules that automatically select the difficulty level matching the patients' abilities. The results section shows the validity of the hypothesized recovery strategy and how this behavior was used to tune the algorithm. The discussion and conclusions discuss the findings, acknowledge the limitations and propose possible extensions of the study.

\section{ReCOVERy StRategy HyPOTHESIS}

In patients after stroke it is assumed that there is a large interindividual variability in the capacity to recover [27]. The majority of patients recover proportionally from impairment and after about six months the impairment reaches a plateau. In addition, patients with chronic stroke who seek further treatment can be subdivided into two categories: those with severe initial impairment who recover to moderate levels of impairment and those who remain severely impaired [27]. In order to precisely qualify and quantify the different components of motor recovery in a group of stroke patients treated by robot-aided techniques, we recently proposed the use of a robot measured evaluation method that includes measurement of the efficacy, efficiency, accuracy, mean speed, smoothness and force control components of movement [28]. In particular, we postulated that knowledge of the recovery components and of the associated performance acquisition model should make it possible to precisely plan and, if necessary, modify the rehabilitation strategies and use this information to accelerate the processes of motor relearning. Furthermore, we analyzed the improvements of movement dynamics and kinematics during robot-assisted rehabilitation in a group of chronic stroke individuals and assessed the time course of recovery over three weeks of training [29]. The results showed that the force control parameters and the movement smoothness improved quickly at first and then plateaued, while steady gains in mean velocity of movement took place over a longer time course. These findings led us to hypothesize the presence of a recovery strategy in which different concurrent mechanisms with diverse time constants contribute to the neural adaptation resulting from the robotic training.

On the other hand, experiments of motor adaptation to a force field of the lower limb in normal subjects have shown that the motor adaptation can be modelled as a process in which the motor system greedily minimizes a cost function of kinematic error and effort [30]. Therefore, we refined our hypothesis postulating that the performance improvement process in our patients could be considered as the result of different concurrent optimization processes including adaptation of both kinematics and effort parameters, starting at the same time but having different time constants. In practice we used this hypothesis to choose the subset of parameters estimating patients' performance to be included in the algorithm and to design the set of rules to identify the plateau of adaptation in the parameters (steady performance) to optimize training.

\section{Methods}

\section{A. Subjects}

The recovery strategy hypothesis was tested in a group of 22 patients after stroke ( 7 females and 15 males; age $53 \pm 11$ ). 
The study was performed at the Salvatore Maugeri Foundation, IRCCS Rehabilitation Institute of Veruno (Veruno, NO, Italy). All patients were at a chronic stage, their unilateral cerebrovascular accident (CVA) having occurred at least six months prior to enrolment ( $32 \pm 42$ months from CVA). Inclusion criteria were the presence of a single unilateral CVA and the presence of at least $10^{\circ}$ of motion in the treated joints (shoulder and elbow). Subjects with severe sensory and visual field impairment and aphasia were excluded. Patients had severe to mild impairment (Fugl - Meyer $=20.3 \pm 8.5)$. The study was carried out in conformity with the Declaration of Helsinki of the World Medical Association; all patients gave their informed consent to participate in the study, which had been approved by the local scientific and ethics committees.

In addition, the developed algorithm was tested in closed loop condition, in a sample of five patients after stroke at chronic stage enrolled with the same criteria reported above.

\section{B. Training Apparatus and Experimental Protocol}

The two DoF elbow-shoulder manipulators MEMOS [31] and "Braccio di Ferro" [32] were used for the treatment of patients involved in the open loop testing. The algorithm was actually implemented, and tested in the closed loop condition, only for the "Braccio di Ferro" device. The robot apparatus included an end-effector, normally consisting of a sensorized handle which is grasped by the patient and moved through the workspace of the device (i.e., in the horizontal plane). The control strategies implemented, enabled subjects to execute both completely voluntary movements and 'shared' controlled movements in which the device helped the subject to carry out the part of the task he/she was not able to do autonomously. Patients had their trunk fastened to the back of the chair by a special jacket to limit compensation phenomena and were placed at the robot desk facing a video screen that provided visual feedback of the assigned motor task. The patient's paretic limb was supported at the elbow by a low friction pad that slid along the surface of the robot workspace. Patients had to make a sequence of point to point reaching movements in the shape of a geometrical figure; a yellow circle displayed on the screen indicated the task's starting position, a red circle the task's target position, and a green circle the current position of the handle. A practice session preceded the treatment, during which detailed instructions were given to shorten the exercise learning phase. During the exercise, the device stored details of the handle positions and device status, reporting information about the different robot conditions (patient active, robot active, rest, etc.) acquired at a rate of $100 \mathrm{~Hz}$.

All patients, including the five enrolled in the closed loop experiments, were trained twice a day, five days a week for at least three weeks. Each training session consisted of four cycles of exercise lasting $5 \mathrm{~min}$ each followed by a 3 min resting period. The robot session was fully supervised by the therapist only during the learning phase. Following this, supervision was limited to the patient's connection and disconnection to the device. Before the beginning of a new training session the therapist monitored the patient's global performance and, in the case of quasi optimal behavior (subjectively evaluated), changed the difficulty level of the motor task by selecting a more complex reaching sequence. In the closed loop test the difficulty level of the task was automatically adjusted by the algorithm based on the patient performance computed data. During these experiments the therapist was requested to independently judge the patient's behavior and agree/disagree with the algorithm decision. Details about this procedure will be given later. At the end of training, feedback on how the five patients perceived the training was obtained by means of a reduced version of the Intrinsic Motivation Inventory (IMI) questionnaire [33].

On the same days as robot treatment, all patients underwent physical therapy performed by professionals according to the Italian Stroke Prevention and Educational Awareness Diffusion (SPREAD) guidelines for 45 min a day.

\section{Design of the Progressive Task Regulation (PTR) Algorithm}

The basic idea behind the PTR algorithm is to promote patients' motivation and the generalization processes through varied therapy practice in the widest spectrum of patients as possible. For this reason we designed the algorithm to provide different types of assistance and tasks. To be an efficacious motor task an exercise must be motivating, i.e., it should have a difficulty level that allows the patient to execute at least part of the assigned motor task, at the same time continuously challenging the limits of the patient's disability and trying to overcome them. Therefore, we generated a list of motor tasks with increasing difficulty level and designed the algorithm to regulate training by selecting the task and assistance that is most suitable for the patient's current ability, based on the obtained performance. This approach should overcome the shortcomings of previously presented algorithms that aim only to optimize the level of assistance.

\section{Difficulty Level of the Motor Task}

Usually a motor task is characterized by a set of features determining the so-called "difficulty level of the task"; a change of these features usually produces an increase/decrease of the task's difficulty. In particular, we have identified the following features.

1) Reaching Task Sequence: In practice the exercise is similar to the tracing of a geometric shape. These shapes have increasing number of edges and complexity: a square represents the easiest reaching task, an eight-directions reaching task (involving the training of different muscle synergies) the most difficult.

2) Type of Assistance: In the present robot version, we decided to maintain the maximum force magnitude assisting the patient movements at a predefined value for all the difficulty levels of the task. Therefore, the algorithm can only change the time of its actuation and its direction (assistive or repulsive force). Based on these conditions, the system we implemented is able to provide three types of assistance (Fig. 1).

Time-Triggered Assistance (TTA): This type of assistance is used in the more compromised patients. At the beginning of the task the patient is free to move the arm within the workspace, but $2 \mathrm{~s}$ after visualization of the target on the robot display the assistive force comes into play at a very low magnitude. The assistance is then gradually increased in ramp-like mode up to a predefined value (soft application of assistance). The force is 


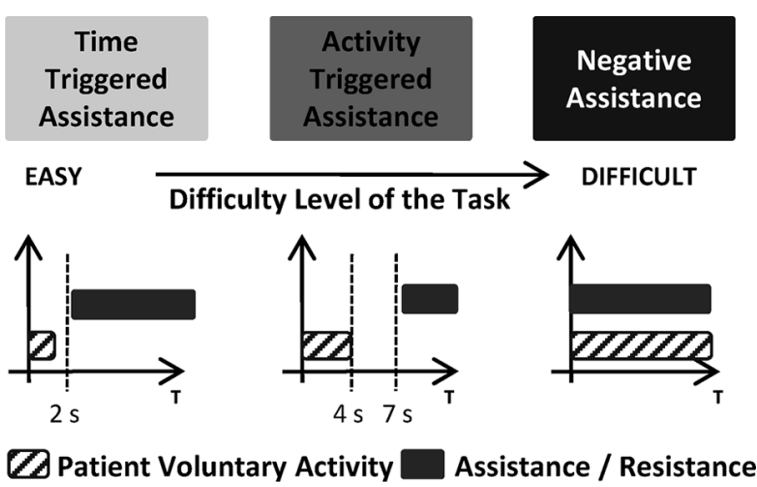

Fig. 1. Types of assistance implemented in the controller of the robotic device. Left: TTA. Middle: ATA. Right: NA. The grayed and dashed areas indicate, respectively, the typical time course of actuation of assistance and the patient's voluntary activity.

then maintained at that magnitude so as to guide the patient's arm to the target. In this way the patient is challenged to independently begin the motor task but at the same time helped in any case to complete the movement, so motivating the patient to perform the exercise.

Activity-Triggered Assistance (ATA): This type of assistance has the objective of stimulating and enhancing the patient's voluntary motor activity. The patient is requested to move the arm from the starting point to the target, the only restriction being obviously the horizontal plane supporting the arm. If during the motor task execution the patient cannot complete the task autonomously, the robot evaluates the current position of the end-effector and, after a period of $3 \mathrm{~s}$ in the same place, guides the patient's arm to the target position.

Negative Assistance (NA): In this type of assistance, the patient is required to execute the reaching task, working against a resistive force of constant magnitude. This task is designed for less compromised patients or for those who have already made a significant motor recovery and need mainly to improve the quality of their motor control.

The fact of providing three different types of assistance should increase the number of patients who can benefit from robotic treatment.

3) Target Distance: The distance between the starting position and the target can be selected from two options: 150 and 220 $\mathrm{mm}$. An increase of the target distance is usually implemented before any change of the type of shape.

4) Virtual Slot: The learning process occurring during the course of rehabilitation refines the selection and coordination of the appropriate muscular contractions, and implies the creation of new motor synergies by combining forces generated across multiple joints in novel kinematic and dynamic patterns. In order to stimulate the patient to recruit the correct muscle synergies during the movement towards the target, we have introduced a virtual slot $(100 \mathrm{~mm}$ width, centered on the theoretical path). This is a slightly different concept from the virtual slot defined in other devices, because the patient is not constrained to the theoretic trajectory so removing any kinematic error from the movement. Within our virtual slot the patient is free to move anywhere without resistance; conversely, outside the slot, movements are prevented by a high stiffness value. In practice it limits only large trajectory deviations, so giving sense to the computation of performance parameters also in the case of virtual slot. This feature was enabled only in the presence of large accuracy errors.

Different patients usually have different motor skills and different rates of motor learning. Therefore, they exhibit different abilities in the execution of specific tasks with different features. We thus took into account the spectrum of variation of the previously defined features, and defined a list of tasks with increasing difficulty level. This list included, for example, different reaching sequences like drawing a square or more complex geometrical shapes. The task list was submitted to an expert therapist who assigned the difficulty levels from the easiest to the most difficult.

\section{E. Measurement of Patient's Performance}

Before training, the patient undergoes an evaluation session in which, starting from the workspace center, he/she must perform at least two reaching movements in eight specific movement directions $\left(0^{\circ}, 45^{\circ}, 90^{\circ}, 135^{\circ}, 180^{\circ}, 225^{\circ}, 270^{\circ}, 315^{\circ}\right)$. During the evaluation session the device does not generate any assistive force so that only motor behavior due to the patient's voluntary activity is evaluated. During this session the device records the position of the end-effector and computes the following performance parameters [9].

1) Active Movement Index (AMI): the patient's ability in executing the assigned motor task without robot assistance. This index represents the percentage of trajectory travelled by means of the patient's voluntary activity.

2) Mean Velocity (MV): the mean value of the velocity of the end-effector.

3) Normalized Path Length (nPL): obtained by computing the path length of the trajectory travelled by the patient to reach the target and normalized to the theoretical path. This parameter is a measure of the error of movement efficiency; therefore decreasing values during training reflect an improvement of efficiency in the motor task execution. It can be considered also an index of movement effort.

4) Mean Distance (MD): the mean absolute deviation of the points of the patient's path from those of the theoretical path. It is an index of movement accuracy.

After the start of training the above performance parameters are recursively computed at the end of each training session in order to verify if a change in the difficulty level is required.

The performance parameters are always measured during the patient's voluntary activity phase of each reaching movement, and averaged so as to obtain for each parameter one mean value for each training session. When the TTA assistance is selected the algorithm computes only the AMI parameter during the time window without assistance (2 s window).

\section{F. Rules for Difficulty Level Transition}

The PTR algorithm adjusts the difficulty level of the exercise on a session by session basis; this means that patients practice the same task during a therapy session. Fig. 2 presents the flow chart of the procedure implemented. According to the algorithm presented by Li et al. [22] for each parameter the process of 


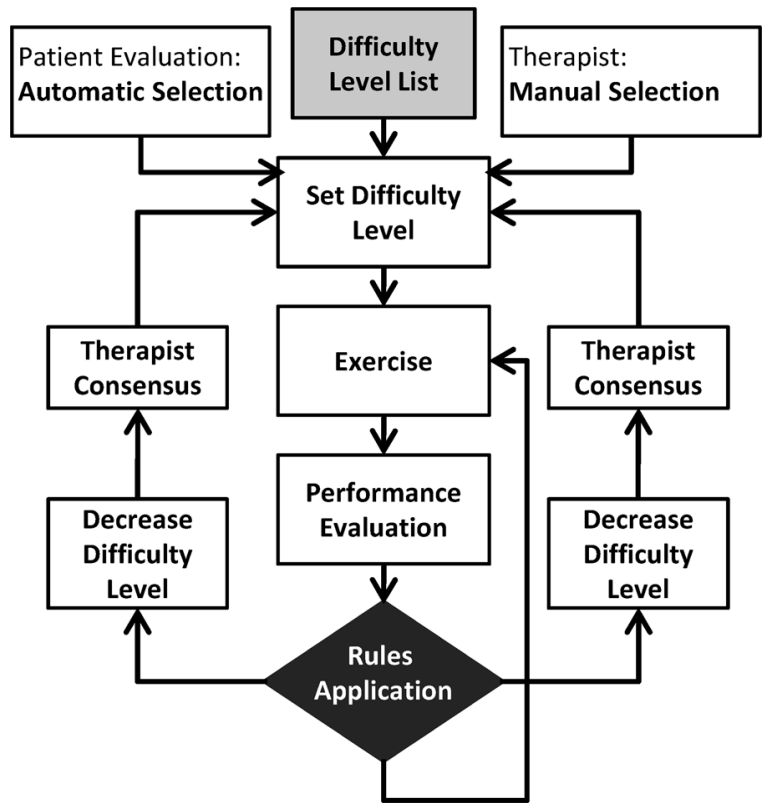

Fig. 2. Flow chart of the procedure implemented in the PTR algorithm. Actual change of the difficulty level is subject to the judgement of the therapist who can decide whether to accept or refuse it.

difficulty level adaptation is controlled by a moving average procedure obtained over three consecutive training sessions.

The TTA is implemented using a single difficulty level with the patient executing the easiest task (a square shaped task, 150 $\mathrm{mm}$ reaching size). If AMI exceeds $20 \%$ then a transition to ATA is proposed. When the difficulty level selected includes ATA or NA assistance then the algorithm applies the following transition criteria.

We defined with $\mathrm{P}_{i}$ the performance vector at the training session $i$

$$
P_{i}=\left[\begin{array}{c}
\mathrm{AMI}_{i} \\
\mathrm{MV}_{i} \\
-\mathrm{nPL}_{i}
\end{array}\right]
$$

where $\mathrm{AMI}_{i}, \mathrm{MV}_{i}$, and $\mathrm{nPL}_{i}$ are the mean values of the previously defined parameters computed at the training session $i$ (average of all the values obtained in every reaching movement of session $i) . \mathrm{nPL}_{i}$ is expressed as a negative value so that increases in the parameter equal increases in performance.

In order to obtain smooth variations of performance, a moving average procedure is applied to the vector over three training sessions, i.e.,

$$
\bar{P}_{i}=\frac{\sum_{k=i-2}^{i} P_{k}}{3} .
$$

Then the performance change is evaluated through the relationship, as shown in (3) at the bottom of the page. where the threshold values are $\alpha=50, \beta=85, \gamma=65, \delta=1.35, \zeta=2$, $\eta=0.016$. Here, we report only their meaning; their determination will be discussed later in the results.

The algorithm works through a thresholding approach on monotonic increase/decrease and detection of the plateau of adaptation of the patient's performance. $\alpha$ is the lower limit of voluntary activity to identify a performance decrease. $\beta$ is the upper limit of voluntary activity to identify a performance increase. The first term in round brackets of the (b) relationship detects a monotonic increase of performance; the second term in round brackets detects plateau of adaptation. In particular $\zeta$ and $\eta$ values are the increment steps to detect a monotonic increase in the $\mathrm{nPL}$ and MV parameters whereas $\delta$ and $\gamma$ are the threshold values to detect a plateau respectively of the same parameters. The matrix notation adopted implies an implicit logical AND condition for the elements of the performance vector. In practice if $\mathrm{PC}=-1$ the patient's performance is worsening, if $\mathrm{PC}=0$ the performance is unchanged, if $\mathrm{PC}=+1$ the performance is improving. Then a performance score is obtained by the performance change value through the following relationship:

$$
\mathrm{SP}_{i}=\left\{\begin{array}{c|c}
-1 & \mathrm{PC}_{i}=-1 \\
0 & \mathrm{PC}_{i}=0 \\
\mathrm{SP}_{i-1}+1 & \mathrm{PC}_{i}=+1
\end{array}\right\} .
$$

Finally, the difficulty level of the next task is determined based on the performance score through the following relationship:

$$
\mathrm{DL}_{i+1}=\left\{\begin{array}{c|c}
\mathrm{DL}_{i}-1 & \mathrm{SP}_{i}=-1 \\
\mathrm{DL}_{i} & 0 \leq \mathrm{SP}_{i} \leq 2 \\
\mathrm{DL}_{i}+1 & \mathrm{SP}_{i}=3
\end{array}\right\} .
$$

After a change of difficulty level the scoring performance $\mathrm{SP}_{i}$ is reset to zero.

In practice, for a difficulty level increase it is required that AMI exceeds a threshold value and MV and -nPL exceed a threshold value or exhibit a monotonic increase over three consecutive sessions. Conversely for a difficulty level decrease it is required that the AMI goes below a threshold value (different from the previous) and MV and -nPL exhibit a monotonic decrease over one training session. This is to prevent the patient working for a long period at an unsuitable difficulty level of the task.

In addition the algorithm allows the inclusion of a virtual slot in order to prevent the patient working in workspace positions far from the theoretical trajectory (the straight line between the

$$
\mathrm{PC}_{i}=\left\{\begin{array}{c|cc}
-1 & \bar{P}_{i}<\left[\begin{array}{lll}
0 & 0 & 0 \\
0 & 1 & 0 \\
0 & 0 & 1
\end{array}\right] & \bar{P}_{i-1}+\left[\begin{array}{c}
\alpha \\
0 \\
0
\end{array}\right] \\
0 & S_{a} \wedge S_{b}=\{\} \text { with } S_{a}, S_{b}, \text { solution set of (a) and (b) } & (a) \\
1 & \left(\bar{P}_{i}>\left[\begin{array}{lll}
0 & 0 & 0 \\
0 & 1 & 0 \\
0 & 0 & 1
\end{array}\right]\right. & \left.\bar{P}_{i-1}+\left[\begin{array}{c}
\beta \\
\zeta \\
\eta
\end{array}\right]\right) \vee\left(\bar{P}_{i}>\left[\begin{array}{c}
\beta \\
\gamma \\
\delta
\end{array}\right]\right)
\end{array}\right\}
$$



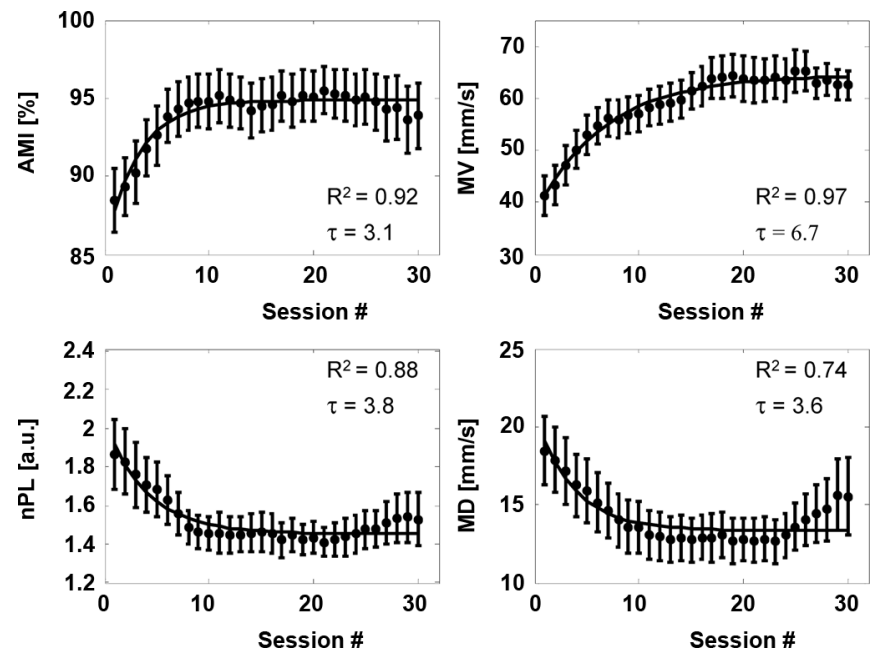

Fig. 3. Values of the AMI, MV, nPL, and MD parameters over the course of 32 training sessions of therapy in the group of 18 patients after stroke who did not exhibit the hierarchy phenomenon. Each point represents the average value obtained by the group for each training session. The vertical bars represent the standard error. The overlapping curve is the exponential increasing/decaying model fitting the parameter's values obtained during the training.

starting point and the target). The virtual slot is always active during TTA and switched-off during NA. During ATA, it is automatically activated/deactivated, independently of the reaching sequence selected, based on the value assumed by the MD parameter averaged over three training sessions through the following relationship:

$$
\mathrm{VS}_{i+1}=\left\{\begin{array}{l|l}
1 & \overline{\mathrm{MD}}_{i}>\varepsilon 1 \\
0 & \overline{\mathrm{MD}}_{i}<\varepsilon 2
\end{array}\right\}
$$

where $\varepsilon 1=50 \mathrm{~mm}$ and $\varepsilon 2=40 \mathrm{~mm}$. The actual change of the difficulty level of the task is in any case subject to the judgement of the therapist who can decide whether to accept the automatically suggested change or refuse it (leaving the patient working in the same conditions).

\section{RESULTS}

\section{A. Patient's Recovery Strategy Assessment}

Fig. 3 reports the time course of AMI, mean velocity, nPL, and MD parameters during 32 training sessions in 18 chronic patients who exhibited a clear exponential decreasing effort pattern right from the outset of training. Each point represents the average value obtained by the group for each training session. The vertical bars represent the standard error. It can be seen that the average acquired performance follows an increasing/decreasing exponential law that allows the computation of the time constant related to the exponential increase/decay. The time constant was 3.1 sessions for AMI, 3.8 sessions for nPL, 3.6 sessions for MD, and 6.7 sessions for MV.

The MV time constant was significantly different from AMI $(p<0.001)$ and MD $(p<0.01)$ time constants (unpaired $\mathrm{t}$ test). The plateau was reached after about 10 training sessions (one week of training) for AMI, nPL and MD, and after about 20 training sessions (two weeks of training) for MV. The fitted
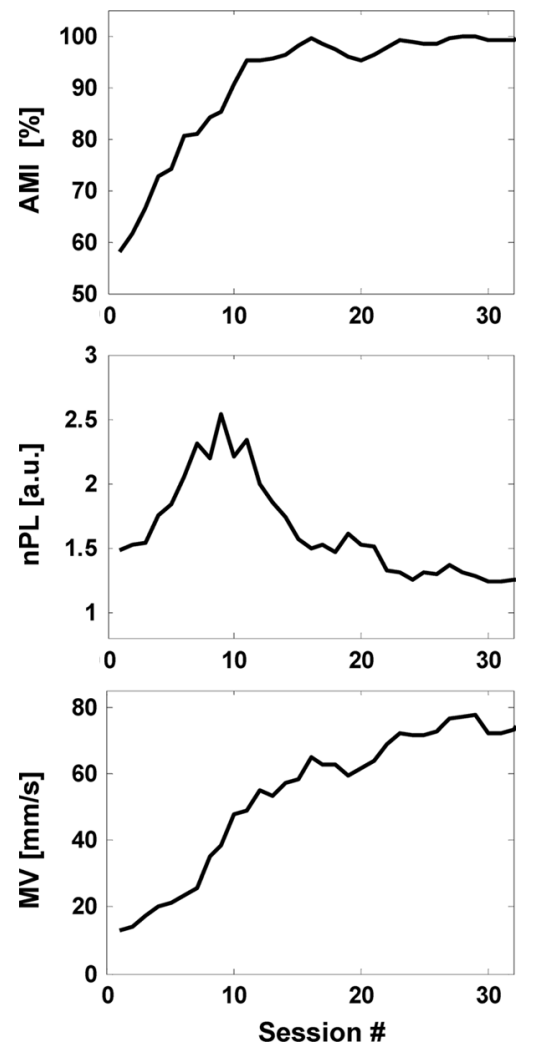

Fig. 4. Time course of recovery observed in a severely impaired chronic stroke patient. Top: AMI parameter representing the patient's voluntary activity during training. Middle: nPL parameter representing the effort (energetic cost) exerted by the patient-it clearly evidences the presence of a hierarchy phenomenon. Bottom: MV parameter, continuously improving during training.

models had a very high correlation with the data $\left(\right.$ AMI $\mathrm{r}^{2}=$ $\left.0.92 ; \mathrm{MVr}^{2}=0.97 ; \mathrm{nPL} \mathrm{r^{2 }}=0.88 ; \mathrm{MD} \mathrm{r}^{2}=0.74\right)$. Based on the different time constant values we hypothesized the presence of a three-step recovery strategy, in which the patient: 1) tried to achieve the goal, 2) optimized the effort, and 3) increased movement speed. Of course these are concurrent processes starting at the same time and each having a different dynamic evolution; the former faster and the latter slower.

Fig. 4 shows the time course of the AMI, nPL, and mean velocity parameters of a patient during training with the robotic device. We observed the presence of a hierarchy phenomenon in the nPL parameter. In fact, the effort was low at the start of training because most of the task was executed with robot-assistance; then the effort gradually increased to reach a maximum value as voluntary activity increased. At $85 \%$ AMI the effort decreased again and reached a plateau. This particular pattern of effort adaptation was evident only in four of the 22 patients; three of them are reported in Fig. 5. They exhibited a clear peak in the effort parameter around the level of $80 \%-85 \%$ of AMI but with different delays from the training start. These four patients had a high impairment FM pretreatment $=12,15,9,28)$. The remaining 18 patients exhibited an effort pattern similar to that presented in Fig. 5(a). Even if the hierarchy phenomenon was present only in $18 \%$ of our patients we took into consideration also this type of behavior to customize the algorithm (see next section for details). 

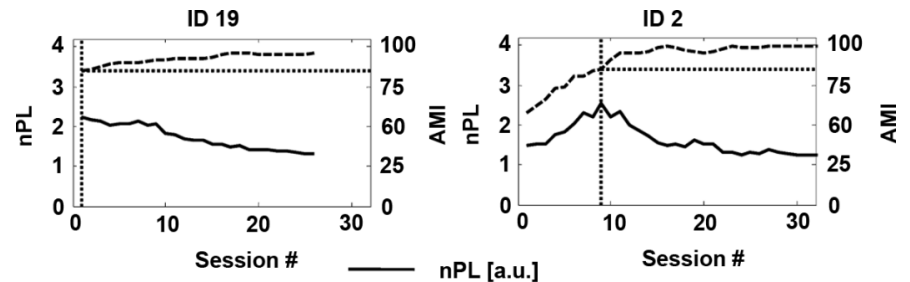

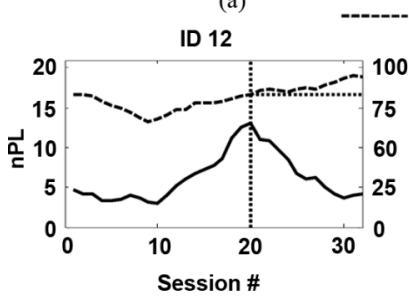

(c)

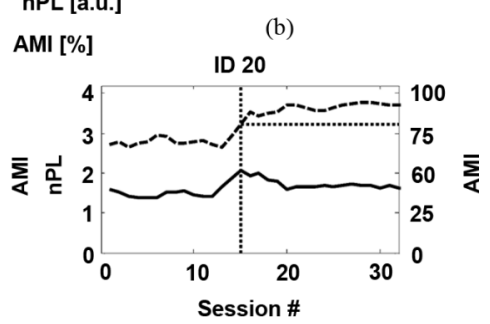

(d)
Fig. 5. Time course of recovery over 32 training sessions of the AMI and nPL parameters. Panel (a) presents a patient in whom the decay of the nPL starts from the beginning of training. Panels (b)-(d) present three examples of the hierarchy phenomenon observed in 4 out of 22 patients. It is worth noting that the peak observed in the nPL is obtained when the AMI reaches a $80 \%-85 \%$ level of voluntary activity. Hierarchy peaks had different amplitudes and times (session) of occurrence during training.

\section{B. PTR Algorithm Tuning}

The PTR algorithm was tuned based on the results of patients' recovery assessment reported above. The use of the parameter $\alpha$ implies that a consistent worsening of performance is required in order to decrease the difficulty level of the task. The $85 \%$ level of the $\beta$ threshold was selected according to the finding previously described for the hierarchy phenomenon (i.e., the voluntary activity level to reach the peak in the effort parameter). This should guarantee that the effort optimization process is started even in the most impaired patients. Subsequently, the PTR algorithm was tuned in order to mimic the behavior of the therapists in selecting the difficulty level of the task. In particular, the threshold values $\delta$ and $\gamma$ were selected by using a software procedure that varied the thresholds for $\mathrm{nPL}$ and MV parameters in a predefined range $(1.0-2.0$; step $=0.05$ for $\mathrm{nPL}$ and $40-90$; $\mathrm{step}=2.5$ for MV) and computed at which training session a difficulty level change was suggested by the algorithm. Then we computed the correlation between the training session in which a difficulty level change was actually selected by the therapist and that selected by the automatic algorithm. Fig. 6 shows the resulting surface representing the distribution pattern of the correlation values obtained by this procedure. The surface clearly evidences a peak of correlation $(r=0.81 p<0.001)$ which allows to identify the optimal threshold values for nPL and MV parameters. In practice for these values the algorithm and the therapist exhibited the most similar behavior.

The $\zeta$ and $\eta$ values were selected empirically by setting a minimum $2 \%$ step per session for the detection of a monotonic increase of the parameters. Finally, the $\varepsilon 1$ and $\varepsilon 2$ values were selected by computing the mean value and standard deviation of MD at the third training session $(18 \pm 9 \mathrm{~mm})$ in the group of 22 poststroke patients and selecting approximately the mean plus, respectively, the 2 and 3 SD levels.

After the tuning procedure in six subjects both the therapist and the algorithm did not suggest any change. In two subjects

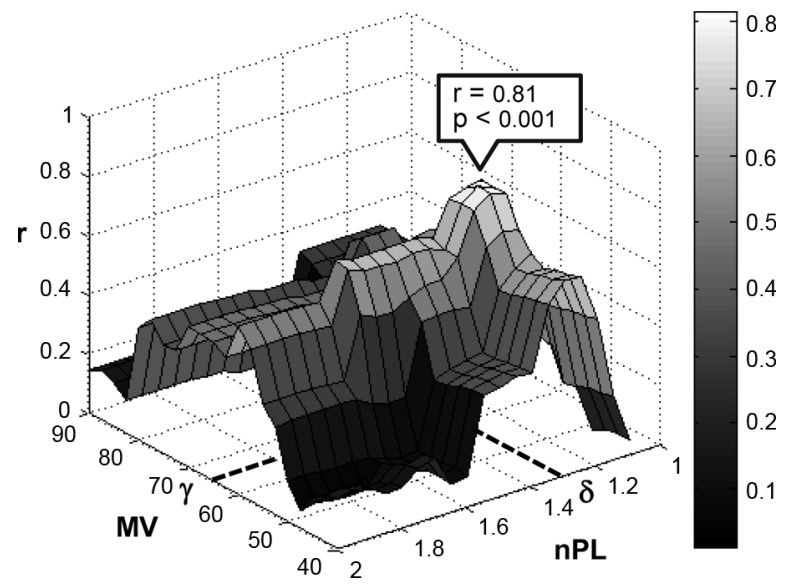

Fig. 6. Pattern of distribution of the correlation values between the training session of difficulty level change selected by the therapist and that selected by the automatic algorithm. The pattern was obtained by applying the algorithm to the performance data obtained in 22 chronic stroke patients. In particular, a software procedure varied the thresholds for $\mathrm{nPL}$ and $\mathrm{MV}$ parameters in a predefined range $(1.0-2.0$; step $=0.05$ for $\mathrm{nPL}$ and $40-90$; $\mathrm{step}=2.5$ for $\mathrm{MV})$ and computed at which training session a difficulty level change was suggested by the algorithm. The surface clearly evidences a peak of correlation $(r=0.81$; $p<0.001$ ) which allows to identify the optimal threshold values for nPL and MV parameters.

TABLE I

PTR Algorithm Performance In the Closed Loop Test

\begin{tabular}{cccc}
\hline \hline $\begin{array}{c}\text { Patient } \\
\text { ID }\end{array}$ & $\begin{array}{c}\text { PTR } \\
\text { Algorithm } \\
\text { Session of } \\
\text { Change }\end{array}$ & $\begin{array}{c}\text { Therapist } \\
\text { Judgement }\end{array}$ & $\begin{array}{c}\text { Starting type } \\
\text { of Assistance }\end{array}$ \\
\hline CL 1 & 8 & agreement & Time Triggered \\
CL 2 & 5 & agreement & Time Triggered \\
CL 3 & 12 & disagreement* & Activity triggered \\
CL 4 & 6,14 & agreement & Activity triggered \\
CL 5 & 6 & agreement & Time Triggered \\
\hline \hline
\end{tabular}

*change forced by the therapist

the therapist changed the difficulty level in advance of the algorithm. In one subject, the therapist did not change the diffculty level and the algorithm changed two sessions before the end of training. In six subjects, the algorithm changed the difficulty level consistently in advance, demonstrating a greater sensitivity than the therapist.

\section{Closed Loop Testing of the PTR Algorithm}

Table I reports the results obtained by applying the PTR algorithm in closed loop conditions, i.e., the changes were suggested by the algorithm during training and submitted to the therapist's judgement. Note that in this case the behavior of the algorithm could influence the patient's outcome. The first column reports the session number of automatic difficulty change, the second column the therapist agreement/ disagreement and the third column shows the starting type of assistance of the practiced motor tasks.

In only one subject did the therapist not agree with the algorithm proposal and forced a difficulty level change in advance of the algorithm.

On average the five patients involved in the closed loop test improved their impairment (FM change) by about 3.0 points. 
TABLE II

SubSCALE FINDINGS OF THE IMI QUESTIONNAIRE EVALUATED IN THE Five PATIENTS ENROLLED IN THE ClOSED LOOP EXPERIMENTS $($ Subscale RANGE $=1-7)$

\begin{tabular}{lc}
\hline \hline \multicolumn{1}{c}{ IMI subscale } & Score \\
\hline Interest/Enjoyment & $5.1 \pm 1.2$ \\
Perceived Competence & $3.6 \pm 1.3$ \\
Effort/Importance & $5.5 \pm 1.4$ \\
Value/Usefulness & $5.2 \pm 1.5$ \\
Pressure/Tension & $3.0 \pm 1.8$ \\
Pain & $1.5 \pm 1.0$ \\
\hline \hline
\end{tabular}

Table II reports the mean values and standard deviations of five preselected subscales of the IMI questionnaire and pain subscales, evaluated in the five patients enrolled in the closed loop experiments. The interest/enjoyment subscale, i.e., a self-report measure of intrinsic motivation, the effort/importance and the value/usefulness of therapy obtained a high score and a low standard deviation. This suggests that our patients found the robot treatment very interesting. The perceived competence and pressure/tension subscales obtained a low-mid score. This is probably due to the fact that the majority of patients were highly impaired. Pain obtained a low score, signifying that most of them did not experience pain during training with the robot device. These results are similar to those we obtained in a previous study of robot-assisted rehabilitation [33].

\section{DISCUSSION}

We have presented here an algorithm able to evaluate patients' performance during training of the upper limb with a robotic device and automatically adapt the difficulty level of the motor task based on the obtained performance. The algorithm was implemented taking into account the findings obtained in a study previously conducted in a group of 22 patients after stroke in chronic condition, by hypothesizing and verifying the presence of a specific recovery strategy during the course of treatment. In practice, the algorithm aims to optimize training when a steady performance is detected, mimicking the behavior of an expert therapist.

We observed in patients the existence of a recovery strategy consisting of concurrent optimization processes such as goal achievement, effort optimization and movement speed improvement, each having a different dynamic evolution as demonstrated by the different time constants; the former faster and the latter slower. Hence, even in chronic poststroke patients the motor adaptation obtained by training seems to be compatible with that presented by Emken et al. [30] in which the motor system of healthy subjects adapted to a force field by minimizing the kinematic error and effort. In particular, assessment of the time course of recovery in our patients showed that most of them improved their efficacy, effort, and accuracy of movement and then over a longer time their movement speed. In addition, a hierarchy phenomenon dependent on the level of voluntary activity (i.e., the patient's ability) was observed in some of the more impaired patients. Even if the limited number of patients studied precludes any significant statistic, it seems that the effort optimization process could start only after a certain level of voluntary activity was reached. Eight other patients who were also highly impaired $(\mathrm{FM}<20)$ did not exhibit the hierarchy phenomenon, but six of them started treatment with AMI $>80 \%$ and two of them could not improve their AMI over $80 \%$ after training.

In the development of this algorithm we sought to answer the question: "what to do when the patient has fully acquired the assigned motor task?". The answer of course is to match the task difficulty to the learner's skill level both initially and as the relearning process progresses [23].

Like other performance-based progressive algorithms presented in the literature [19], [21], the PTR algorithm operation is partly dependent on the type of controller implemented and some hardware features of the robotic device. The devices we used to treat our patients are based on impedance ("Braccio di Ferro") and admittance (MEMOS) controllers to implement the same type of reaching task. Because the algorithm was designed in view of its application with structurally different devices, we decided to base its design only on features related to general principles and matching both the patient's and therapist's behavior. In contrast to the MIT-Manus device, the "Braccio di Ferro" did not include a force sensor; for this reason we decided to estimate effort using an indirect measure based on a kinematic parameter (nPL) providing an estimate of the energetic cost of the patient's behavior. The AMI, providing an index of the patient's voluntary activity, allows both the estimation of the initial (ability to initiate movement) and final conditions (target error) of the movement like the PM1 and PM4 performance measures of the MIT-Manus [19]. In addition, the nPL and MD parameters estimate the performance during the movement and can be considered partly equivalent to the PM2 and PM3 metrics. In fact, in these two performance measures the MIT-Manus includes both kinetic and kinematic information.

What really distinguishes our algorithm from that implemented in the MIT-Manus is that the latter uses the performance information to regulate the assistance delivered to the patients whereas our algorithm actively changes the task and some related features or the type of assistance to increase the difficulty level of the motor task. In addition, the requirement of an improvement in the mean velocity should ensure the consolidation of the learned skill. In a certain sense the two algorithm can be considered complementary, and it is easy to imagine our algorithm as an extension of the other, or, for example, the progressive regulation of assistance as an extension of our time triggered assistance. Both share the philosophy of providing a mechanism that dynamically maintains performance near to an optimal challenge point at which performance gains during learning are maximal. In other words, both provide a method for a patient to evolve from hemiplegic to normal arm movement. In addition, the automatic changes of difficulty level, simulating a video-game experience, may be very useful for maintaining the patient's interest high during the whole training, so inducing better patient motivation and outcome, and promoting generalization processes through varied therapy practice. 
The algorithm proposed by Cameirao et al. [25] includes a personalized training module that autonomously adjusts the difficulty of a virtual reality (VR) game session on a trial by trial basis by capturing specific features of the movement of the arms. This module uses a simple model that checks for two thresholds and acts whenever the patient's performance goes above or below them. Also the PTR algorithm adopted a similar solution, but, in contrast to VR rehabilitation protocols which usually include only mildly impaired patients, we employed a more sophisticated method to evaluate patient's performance and implemented some additional rules to capture the recovery strategy of the most impaired patients.

\section{A. Study Limitations and Future Developments}

The sample of five patients enrolled in the closed loop testing of the algorithm was useful to assess the agreement with the therapist decision and how patients perceived the training, but it is too limited to be able to determine to what extent patients' improvement is better than that obtained without the use of this algorithm. It would be reasonable to think that the outcome obtained with versus without the algorithm is comparable because it should depend mainly on the patient's recovery capacity and training intensity. Conversely, we might expect the recovery process to be faster using the algorithm because of its greater sensitivity in the detection and quantization of performance changes. Of course a carefully designed randomized controlled study in a consistent number of patients is required to confirm these hypotheses.

Additionally, the validation of the procedure using a set of data different from that used to tune the algorithm (an additional group of patients) would have been beneficial. Future lines of research could extend the concepts introduced in this study to robotic devices with a higher number of degrees of freedom and allowing the training in 3-D workspace. In this case the metrics used for performance evaluation and also the remarks about the recovery strategy adopted by patients could be different.

Finally, the use of different types of assistance means that our robots could be applied in a wider spectrum of patients. In fact, the TTA is suitable for more severe impaired patients who exhibit only ability to initiate movement. The negative assistance, on the other hand, should allow to improve the quality of movement in mildly impaired patients. Both these categories are susceptible of further development; in fact, the TTA could be extended using brain-computer interfaces techniques [34] to trigger the robot assistance and the NA could be extended by the use of virtual reality or gaming techniques [24], [35] in order to improve patient engagement and motivation, so resulting in an improved outcome.

\section{CONCLUSION}

The algorithm we developed should be useful for the implementation of training protocols allowing individualized and gradual treatment of disabilities of upper limbs in patients after stroke. It is based on optimization principles of motor learning taking into consideration both patient and therapist behavior, and has the potential to enhance the learning effectiveness of the administered tasks. The availability of this type of system should allow an easier management of the different motor tasks administered during training thereby facilitating the therapist's activity in the treatment of different pathologic conditions of the neuromuscular system.

\section{REFERENCES}

[1] P. Langhorne, J. Bernhardt, and G. Kwakkel, "Stroke rehabilitation," Lancet, vol. 377, pp. 1693-1702, May 2011.

[2] A. Heller, D. T. Wade, V. A. Wood, A. Sunderland, R. L. Hewer, and E. Ward, "Arm function after stroke: Measurement and recovery over the first three months," J. Neurol., Neurosurg., Psychiatry, vol. 50, pp. 714-719, Jun. 1987.

[3] A. Sunderland, D. Tinson, L. Bradley, and R. L. Hewer, "Arm function after stroke. An evaluation of grip strength as a measure of recovery and a prognostic indicator," J. Neurol., Neurosurg., Psychiatry, vol. 52, pp. 1267-1272, Nov. 1989.

[4] P. W. Duncan, L. B. Goldstein, D. Matchar, G. W. Divine, and J. Feussner, "Measurement of motor recovery after stroke. Outcome assessment and sample size requirements," Stroke, vol. 23, pp. 1084-1089, Aug. 1992.

[5] H. I. Krebs, N. Hogan, M. L. Aisen, and B. T. Volpe, "Robot-aided neurorehabilitation," IEEE Trans. Rehabil. Engineering., vol. 6, no. 1, pp. 75-87, Mar. 1998.

[6] M. L. Aisen, H. I. Krebs, N. Hogan, F. McDowell, and B. T. Volpe, "The effect of robot-assisted therapy and rehabilitative training on motor recovery following stroke," Arch. Neurol., vol. 54, pp. 443-446, Apr. 1997.

[7] G. B. Prange, M. J. A. Jannink, C. G. M. Groothuis-Oudshoorn, H. J. Hermens, and M. J. Ijzerman, "Systematic review of the effect of robot-aided therapy on recovery of the hemiparetic arm after stroke," J. Rehabil. Res. Develop., vol. 43, pp. 171-184, Apr. 2006.

[8] G. Kwakkel, B. J. Kollen, and H. I. Krebs, "Effects of robot-assisted therapy on upper limb recovery after stroke: A systematic review," Neurorehabilitat. Neural Repair, vol. 22, pp. 111-121, Apr. 2008.

[9] J. Mehrholz, T. Platz, J. Kugler, and M. Pohl, "Electromechanical and robot-assisted arm training for improving arm function and activities of daily living after stroke," Cochrane Database Systematic Rev. (Online), p. CD006876, 2008.

[10] V. S. Huang and J. W. Krakauer, "Robotic neurorehabilitation: A computational motor learning perspective," J. Neuroeng. Rehabil., vol. 6, p. $5,2009$.

[11] J. Carr, Stroke Rehabilitation : Guidelines for Exercise and Training to Optimize Motor Skill. Edinburgh, NY: Butterworth-Heinemann, 2008.

[12] J. S. Sabari, "Motor learning concepts applied to activity-based intervention with adults with hemiplegia," Am. J. Occupat. Therapy, vol. 45, pp. 523-530, Jun. 1991.

[13] N. Maclean, P. Pound, C. Wolfe, and A. Rudd, "Qualitative analysis of stroke patients' motivation for rehabilitation," $B M J$, vol. 321, pp. 1051-1054, Oct. 2000.

[14] N. Hogan, H. I. Krebs, B. Rohrer, J. J. Palazzolo, L. Dipietro, S. E. Fasoli, J. Stein, R. Hughes, W. R. Frontera, D. Lynch, and B. T. Volpe, "Motions or muscles? some behavioral factors underlying robotic assistance of motor recovery," J. Rehabil. Res. Develop., vol. 43, pp. 605-618, Sep. 2006.

[15] P. S. Lum, C. G. Burgar, and P. C. Shor, "Evidence for improved muscle activation patterns after retraining of reaching movements with the mime robotic system in subjects with post-stroke hemiparesis," IEEE Trans. Neural Syst. Rehabil. Eng., vol. 12, no. 2, pp. 186-194, Jun. 2004

[16] R. Riener, L. Lünenburger, S. Jezernik, M. Anderschitz, G. Colombo, and V. Dietz, "Patient-cooperative strategies for robot-aided treadmill training: First experimental results," IEEE Trans. Neural Syst. Rehabil. Eng., vol. 13, no. 3, pp. 380-394, Sep. 2005.

[17] J. L. Patton, M. E. Stoykov, M. Kovic, and F. A. Mussa-Ivaldi, "Evaluation of robotic training forces that either enhance or reduce error in chronic hemiparetic stroke survivors," Experimental Brain Research. Experimentelle Hirnforschung. Expérimentation Cérébrale, vol. 168, pp. 368-383, Jan. 2006.

[18] B. S. Bell and S. W. J. Kozlowski, "Adaptive guidance: enhancing selfregulation,knowledge and performance in technology based training," Personnel Psychol., vol. 55, pp. 267-306, 2002.

[19] H. I. Krebs, J. J. Palazzolo, L. Dipietro, M. Ferraro, J. Krol, K. Rannekleiv, B. T. Volpe, and N. Hogan, "Rehabilitation robotics: Performance-based progressive robot-assisted therapy," Auton. Robots, vol. 15, pp. 7-20, 2003. 
[20] D. Reinkensmeyer, D. Aoyagi, J. Emken, J. Galvez, W. Ichinose, G. Kerdanyan, J. Nessler, S. Maneekobkunwong, B. Timoszyk, K. Vallance, R. Weber, R. de Leon, J. Bobrow, S. Harkema, J. Wynne, and V. Edgerton, "Robotic gait training: toward more natural movements and optimal training algorithms," in IEEE Eng. Med. Biol. Soc. Conf. Proc., 2004, vol. 7, pp. 4818-4821.

[21] M. K. O’Malley, A. Gupta, M. Gen, and Y. Li, "Shared control in haptic systems for performance enhancement and training," J. Dynam. Syst., Measur., Control, vol. 128, pp. 75-85, Mar. 2006.

[22] Y. Li, J. C. Huegel, V. Patoglu, and M. K. O’Malley, "Progressive shared control for training in virtual environments," in World Haptics 2009-3rd Joint EuroHaptics Confe. Symp. Haptic Interfaces Virtual Environ. Teleoperator Syst., Salt Lake City, UT, 2009, pp. 332-337.

[23] Y. Choi, F. Qi, J. Gordon, and N. Schweighofer, "Performance-based adaptive schedules enhance motor learning," J. Motor Behav., vol. 40, pp. 273-280, Jul. 2008.

[24] M. S. Cameirão, S. B. I. Badia, E. D. Oller, and P. F. M. J. Verschure, "Neurorehabilitation using the virtual reality based rehabilitation gaming system: Methodology, design, psychometrics, usability and validation," J. Neuroeng. Rehabil., vol. 7, p. 48, 2010.

[25] Y. Choi, J. Gordon, D. Kim, and N. Schweighofer, "An adaptive automated robotic task-practice system for rehabilitation of arm functions after stroke," IEEE Trans. Robotics, vol. 25, no. 3, pp. 556-568, Jun. 2009.

[26] A. Panarese, R. Colombo, I. Sterpi, F. Pisano, and S. Micera, "Tracking motor improvement at subtask level during robot-aided neurorehabilitation of stroke patients," Neurorehabilitation Neural Repair, Feb. 2012.

[27] S. Prabhakaran, E. Zarahn, C. Riley, A. Speizer, J. Y. Chong, R. M. Lazar, R. S. Marshall, and J. W. Krakauer, "Inter-individual variability in the capacity for motor recovery after ischemic stroke," Neurorehabil. Neural Repair, vol. 22, pp. 64-71, Feb. 2008.
[28] R. Colombo, F. Pisano, S. Micera, A. Mazzone, C. Delconte, M. C. Carrozza, P. Dario, and G. Minuco, "Assessing mechanisms of recovery during robot-aided neurorehabilitation of the upper limb," Neurorehabil. Neural Repair, vol. 22, pp. 50-63, Feb. 2008.

[29] R. Colombo, I. Sterpi, A. Mazzone, C. Delconte, G. Minuco, and F. Pisano, "Measuring changes of movement dynamics during robot-aided neurorehabilitation of stroke patients," IEEE Trans. Neural Syst. Rehabil. Eng., vol. 18, no. 1, pp. 75-85, Feb. 2010.

[30] J. L. Emken, R. Benitez, A. Sideris, J. E. Bobrow, and D. J. Reinkensmeyer, "Motor adaptation as a greedy optimization of error and effort," J. Neurophysiol., vol. 97, pp. 3997-4006, Jun. 2007.

[31] R. Colombo, F. Pisano, S. Micera, A. Mazzone, C. Delconte, M. C. Carrozza, P. Dario, and G. Minuco, "Robotic techniques for upper limb evaluation and rehabilitation of stroke patients," IEEE Trans. Neural Syst. Rehabil. Eng., vol. 13, no. 3, pp. 311-324, Sep. 2005.

[32] M. Casadio, V. Sanguineti, P. G. Morasso, and V. Arrichiello, "Braccio di Ferro: A new haptic workstation for neuromotor rehabilitation," Technol. Health Care, vol. 14, pp. 123-142, 2006.

[33] R. Colombo, F. Pisano, A. Mazzone, C. Delconte, S. Micera, M. C. Carrozza, P. Dario, and G. Minuco, "Design strategies to improve patient motivation during robot-aided rehabilitation," J. Neuroeng. Rehabil., vol. 4, pp. 3-3, 2007.

[34] D. Broetz, C. Braun, C. Weber, S. R. Soekadar, A. Caria, and N. Birbaumer, "Combination of brain-computer interface training and goaldirected physical therapy in chronic stroke: A case report," Neurorehabil. Neural Repair, vol. 24, pp. 674-679, Sep. 2010.

[35] M. S. Cameirão, S. B. I. Badia, E. D. Oller, and P. F. M. J. Verschure, "The rehabilitation gaming system: A review," Studies Health Technol. Informat., vol. 145, pp. 65-83, 2009.

Authors' photographs and biographies not available at the time of publication. 